“ (C) 2019 IEEE. Personal use of this material is permitted. Permission from IEEE must be obtained for all other uses, in any current or future media, including

reprinting/republishing this material for advertising or promotional purposes, creating new collective works, for resale or redistribution to servers or lists, or reuse of any copyrighted component of this work in other works." 


\section{Toward an active aging society: an IT model to engage the aging population}

\author{
Tuan V Ha \\ School of Electrical and Data \\ Engineering \\ University of Technology Sydney \\ Sydney, Australia \\ TuanVinh.Ha@student.uts.edu.au
}

\author{
Doan B Hoang \\ School of Electrical and Data \\ Engineering \\ University of Technology Sydney \\ Sydney, Australia \\ Doan.Hoang@uts.edu.au
}

\begin{abstract}
Many countries around the world are expecting a growing number of elderly people as the society is aging over time. This shift is expected to create a large impact on our health and social security system. The cost of having an increasing proportion of elderly people is emerging as a challenge for governments, so much that our government is encouraging people to stay in the workforce longer. As a result, the aging population requires a solution that allows them to remain productive and keeping them mentally healthy. Existing solutions rely on the benefits of social networks or service networks to keep them active and improve mental health. However, these solutions fail to allow elderly people to act as a value contributor for the society. This paper proposes the design of a new model that allows elderly people to actively and collaboratively provide value to the society through an assistive platform that integrates a service network with a social network. This model combines the advantages of the social network to connect them and utilize the advantages of the service network to create opportunities for elderly people to offer their skills and knowledge to exchange benefits with other users. The proposed model can be used as a mean to engage seniors to the community, allowing them to generate value for themselves and the community while staying mentally healthy.
\end{abstract}

Keywords_- aging; population; service; social; network

\section{INTRODUCTION}

An aging population is a growing concern for many countries. Similar to other countries, Australia has a rapidly aging population, this is due to the effects of people living longer and having fewer children. Migration also has an impact on aging, but its effect is minor [1]. Aging is predicted to accelerate in the short term attributed to the flow of baby boomers. The proportion of working age population is expected to fall, by 2050 , only 2.7 people of working age to support each Australian aged 65 year and over [2]. By 2060 , there will be 25 centenarians for every 100 babies, where currently there is about one centenarian for every 100 babies. As a matter of fact, the Australian government will face more pressure on their budget, mainly reflecting the increase in expenditure on health, aged care and the Age Pension [3]. Apart from that, the term 'Demographic time bomb' is often used in newspaper or reports in many countries to express their concern regarding this matter.

Longer life expectancy and an aging population create a pressure to extend the working lives. This pressure is the result of governments who see an economic imperative to retain older people in the workforce longer, from individual who reaches retirement age but with a high level of health and skills, and from those who want to continue working in some form other than the traditional employment. As a result, the retirement age is due to rise from 65 to 67 in many countries to cope with this demand. However, the level of workforce participation for seniors is varied across different industries. Industries such as child care, construction, etc. may not be suitable for seniors as they involve a lot of physical work.

While many elderly people can enjoy their retirement years, other elderly people do not experience the same way. A recent news [4] reported that many seniors are taking their own lives due to depression and social isolation. This social isolation occurs as the result of the loss of social connection when they withdraw from work. Apart from that, the Western culture also treats older people in a way that aging parents are often not living together with their children. Subsequently, this factor helps promote social isolation. Another factor that contributes to the social isolation issue is that elderly people feel they have lost the ability to contribute to the society [4] and a sense of worthlessness is accumulated as a result. Therefore, social connection and active engagement in the community is arguably the best method for promoting active aging.

There are many platforms that allow elderly people to meet and socialize with each other such as Facebook, Twitter, etc. These platforms operate based on the idea of a social network where users can maintain contact with each other. While social network has the potential to help seniors maintain contact and relationship, their online presence is quite low. Even for the social network developed specifically for them [5]. This happens as they often fail to see seniors as active contributor and many elderly people do not see the benefits in using it [6]. Apart from social networks, there are many service networks that allow their users to host their own services. Some examples of these services network are Ebay, Gumtree, etc. Nevertheless, these types of platforms do not provide enough benefits to encourage their users to connect socially once the transaction is completed. As a result, to keep them active and maintain well-being, the challenges are to design an interactive system that not only allow them to maintain connections with each other but also engage them with other people around as active contributors.

Our solution incorporates a social network with a service network and allows elderly people to act as the value creator for the community. The social network component allows them to maintain or form new social connections with family or friends. The service component allows them to use their skills and knowledge to generate benefit for them, keeping them active and healthy. To attract people, our platform allows them to earn different rewards, from monetary to nonmonetary. Their contribution is recognized by given them different advantages which promote a sense of being valued and encourage continuous participation. Finally, their interest 
is served by a module which proposes to them many services to keep them active and to expand their social interaction. The proposed design can be adopted as a mean to facilitate and harvest of elderly people expertise to provide services that provide value for themselves and the economy.

The remaining of the paper is organised as follows. Section II discusses the related work. Section III focus on our design approach. Section IV shows the results. Finally, Section $\mathrm{V}$ gives the discussion, the conclusion and suggestion on future work.

\section{RELATED WORK}

This section described the related work in this area

To promote active aging, [7] develop a paper-based social network application. The application is called TLATOSKETCH and act as a Facebook client where it allows the user to interact with Facebook through a digital pen and paper. This approach is effective in bridging the gap of IT for elderly people. The project produces many suggestions for improving social interaction and keeping seniors active while aging. However, there are many limitations exist in this study. The project requires many modules to be fully operational. Moreover, extra training is required for elderly people to become familiar with the use of digital pen, particularly for those who experience difficulties in motor control.

To help seniors find a volunteering opportunity that they are interested in, [8] develops a platform to engage elderly people to the community in Taiwan. The platform is aiming at recommending volunteering opportunity to elderly people so that they can be kept active and therefore, reduce social isolation. This is effective as it reinforces the idea that participation in volunteer activities have a positive impact on mental health [9]. The project establishes many valuable considerations such as time, location, etc. when implementing an algorithm that recommends volunteer opportunity to users. These factors help better match the volunteer with a volunteering organization.

To help improve elderly people quality of life and health, [10] develops a platform called AMCOSOP. The platform allows its users to maintain connections with their friends, relative, etc. through a social network platform. The project is effective in addressing the communication needs of elderly people. However, it is not effective in allowing them to act as a value contributor to the society provided that many elderly people are still healthy and possess many valuable skills.

To help elderly people strengthen communication and improve their health condition, [11] propose a senior social network (SSN) with the aim to digitally include all seniors regardless of their educational level or technical skills. The project establishes many valuable considerations regarding the technical difficulty that elderly people face. However, this project also fails to recognize elderly people as active service contributor where they can still contributor more to the community using their skill and expertise.

In addition to the literature attempt to keep elderly people active while aging, there are many ongoing projects in the industry that are aiming to promote active aging in the society. Recently, LinkedIn launched a new feature called Career Advice, a feature that allows its users to act as a mentor for other users. While the feature is not designed specifically for elderly people, it provides a great opportunity for them to engage with people from different generations in the community. Moreover, there are many platforms such as Udemy, Lynda, etc. while these platforms are not aiming at elderly people, they provide a platform for them to use their skills and knowledge to host their classes and generate value for themselves and other people. However, the interaction between students and instructors are limited and therefore are not effective in reducing social isolation. Moreover, elderly people who would like to become an instructor will need to have good technical skills to be able to use video editing software. As a result, these online platforms are not effective for elderly people to reduce social isolation and act as a value creator for the society.

From the above attempts, it is argued that a better solution is required to better engage elderly people to the community, helping them become more active and healthy.

\section{DESIGN AND IMPLEMENTATION}

The design and implementation in the following section were developed as part of the implementation of an online platform that integrated social network and service network for elderly people [12]. It aims to develop a collaborative environment for elderly people and engage them to the community by allowing them to act as a service contributor. While the previous work focuses on proposing the platform and its operation, this paper enhances it by focusing on the design principles that are used to motivate elderly people on the platform, keeping them active on the platform.

\section{A. Design}

To trigger the motivation of our users to provide services, we use a method to engage and motivate elderly people. This method is developed based on different studies [13, 14] on methods to engage elderly volunteer people which focus on these principles:

Reward: They are likely to provide more services if they receive reimbursement for their expenses. For many people, a reward is also a symbolic gesture of appreciation for the work they provided and an indication that their work is of high quality. To accommodate for this principle, users are given the option to charge a certain amount of money as a form of monetary reward and to compensate for their cost of providing services.

Recognition: People often feel happy and valued if they are recognized for the work they have done, particularly for those who have made a significant contribution. To accommodate for this factor, users will be awarded different title depend on their contribution and achievements. The title will help them promote their work to many more users on the platform. As a result, the service provider will have more opportunities to obtain a monetary reward or recognition.

The value of time: In some instances, the amount of time spent on the platform can be viewed as a cost. The obstacles that the users face will not make it worthwhile for them. The main goal of this principle is to minimize the obstacles in completing tasks. As a result, they are likely to spend more time on the platform if it is easy to use and they can understand what needs to be done to accomplish a task. As a result, the usability aspect of the platform is designed to cater to the needs of elderly people such as impaired vision, lack of motor control, etc. 
Social interaction: this is an important factor in the retention of elderly people in many environments, one of the reasons that motivate them to participate in certain activities [14]. For many people, increasing social connection is also a source of satisfaction, a mean to overcome the solitude feeling after they withdrawn from work. This principle is addressed by our social network component which facilitates the interaction between users.

Support: Providing adequate support is a critical factor in increasing level of engagement, particularly for those who lack of knowledge in certain area. For many people, this is an important step in learning new skills in a new environment and enhancing the overall experience.

\section{B. Architecture of the platform}

The technical aspects include the following components:

- Server-side: The main design pattern behind the server side is the ASP.NET MVC using Entity Framework and Repository pattern. This framework helps minimizes the risk of SQL injection or cross-site forgery attack, etc.

- Web client: on the client side, the web application is optimized using Bootstrap so that it is compatible with mobile devices. Along with bootstrap, the platform also uses a mixed approach between single page application and multiple page application to minimize flickering reduce the page load time. The view will only be reloaded if users navigate to a different section on the platform. This method allows users to use the browser back or forward button to move to other sections while also reduce the load time to enhance user experience.

- Third party API: apart from the above design, third-party APIs are used to serve the design of our functionalities. These APIs include Google Firebase Cloud Messaging to provide real-time chat data between users and Google map API to retrieve the location of the proposed service.

The above architecture help minimize the security risks especially for elderly people with limited technical knowledge. Moreover, web application enhances the accessibility of the platform as it can be accessed anywhere without having to search through many applications on the application store. The APIs serve the communication needs and help users' services gain visibility through Google Map

\section{Implementation}

To enable users to acquire the reward, whether it is monetary or non-monetary, the main feature is to allow users to create a service. After the users have created service, it then becomes available for other users to express their interests by booking for the service. Finally, the last step is to deliver the service and receive the reward to compensate for their effort.

- Create service: users who possess an expertise can create a service and decide whether to provide it for free or charge a certain amount to cover their personal cost. A group is also created automatically to facilitate the discussion of the service.

- Booking for a service: users who feel interested in a service can book for that service. After they booked the service, a reference number is given to them and they are added to the group created automatically for that service and contains other users who have the same interest. As a result, users in the group can discuss and share more information and subsequently expand their social connection.

- Delivering of service: before delivering the service, the service provider, who is also the administrator of the group can facilitate the interaction between users. Finally, the service provider can take the discussion from an online environment to an offline interaction. This method can help reduce the awkwardness before the service is delivered.

The above features play an important role in ensuring users are provided a mean to obtain the reward to compensate for their effort and engage with the community.

To help users realize that their contribution is acknowledged, there is a difference between users to indicate their level of participation. Users with a high level of participation help them gain a high level of credibility and allow them to sell their service easier. Apart from that, services that have high review indicate that the service provider has input a considerable amount of time and effort. To help recognize this effort, these services are given a boost in popularity by being promoted throughout the platform.

Our platform provides users with different level ranging from 1 to 10. Each level consists of a short description refers to the level of experience of the user. The more activities they participate in, the higher level they achieve. This way, the platform lets them knows that their contribution is acknowledged. The benefits of achieving higher level include increased credibility and helping their service gain popularity.

Services are promoted throughout the platform based on interest, the level of the users and the rating of the service. The method used to calculate which service will be promoted is as followed:

$$
\mathrm{WR}=(\mathrm{v} \div(\mathrm{v}+\mathrm{m})) \times \mathrm{R}+(\mathrm{m} \div(\mathrm{v}+\mathrm{m})) \times \mathrm{C}+\mathrm{L}
$$

Where:

$$
\begin{aligned}
& \text { WR = Weighted Rating of the service }(\mathrm{WR}) \\
& \mathrm{R}=\text { average review for the service } \\
& \mathrm{v}=\text { number of votes for the service } \\
& \mathrm{m}=\text { minimum votes required to be considered } \\
& \mathrm{C}=\text { the mean vote across the service category } \\
& \mathrm{L}=\text { Level of the user }
\end{aligned}
$$

The above formula is developed using Bayesian rating. The advantage of the above metric as compared to other metrics such as average of the vote, number of vote, etc. is that it balances and smooth out the rating. The parameters used in this formula mainly focuses on the votes of a service. The more good rating a service has, the higher Weighted Rating. This principle acts as a motivational factor for users to provide good and quality service. Another motivational factor is that active users who participate in different activities can gain higher level and subsequently gain higher Weighted Rating as compared to other users. The above features ensure that their effort in participating and 
contributing to the community is well recognized. By giving them some unique advantages over other users, this incentive encourages other users to become more active

It is obvious that users are likely to spend more time on the platform if it is easy to use and does not take too much effort to handle a certain task. Many elderly people developed visual condition that can affect the use of computer such as Presbyopia and Cataract. To allow users to compensate for Presbyopia, most internet browsers such as Chrome and Safari allow users to change the font size and screen magnification. However, these types of adjustments often pose a difficulty if the user is not familiar with the technology. To provide a better experience, the font size on our platform can be easily adjustable. The method to adjust the font size is located at the fixed header bar so it can be spotted an access easily. We provide 3 levels of adjustment. The default of the setting is normal which can be adjusted larger to suit their needs.

Users who develop Cataract may become sensitive to glare and light. As a result, display contrast can be considered too high for some users. Unfortunately, the color design on most of the online platforms are fixed and cannot be changed through the browser setting as in font size. To overcome this issue, we allow users to change the color scheme of the platform. This function is also located at the fixed header bar, so it can be spotted and access easily.

As people get older, the precision of the hand and finger movement start to decrease slowly. This decline indicates that manipulating small object can become difficult. These include moving the mouse pointer to a specific location, clicking on a small object, etc. Since 1950, pointing at objects on a screen follows Fitts' law. Therefore, users can quickly interact with the object if it is large enough. To accommodate for the decline in movement and precision in elderly people, we maximize the clickable area of the target object. This approach is designed using Bootstrap, each block is span between 3 to 6 large columns. This method will ensure the target is not only big enough but also maintain responsiveness if used on mobile device.

Elderly people often suffer from dementia, a form of cognitive impairment that affect their short-term memory capacity and the ability to focus on tasks. As their short-term memory is reduced, they often require detailed steps to follow in completing a complex task. Therefore, lengthy process must be simplified or broken into smaller steps, so they can easily keep track of their progress. Unfortunately, many users need to complete a lengthy process to accomplish a task. This process can be overwhelming which can result in elderly people giving up or start over again. To avoid burdening users' memory, complex tasks on our platform are broken into smaller steps. For instance, our platform divides the registration process into different small interactive questions and present them to the users. The new question will be shown only when they have answered the previous question. Furthermore, their answer is validated straight away after it was provided so they do not need to come back if there is an error.

Traditionally, many platforms include a help page to provide explanation and guidance to assist people in completing certain tasks. However, these help page often involve extra steps in locating them. Our platform uses Bootstrap tooltip to provide explanation at the field level. If the text is too long, a text box containing the guidance is displayed next the field to ensure its visibility. An elderly people friendly User Interface ensure that elderly people, especially those with visual impairment are provided with a smooth and enhanced experience, which in turn save their time and effort in using the platform.

Apart from the User Interface, Privacy setting should also be simplified. On many major social network sites, privacy setting often involve many levels such as setting for personal information, setting for posts, etc. With the many level of settings, older adult can be confused easily. On the other hand, Privacy setting on our platform is also simplified with only one question asking the user who they would like to share their activities with. The option ranges from 'all users' on the platform to 'no one' which is suitable for users who would like to maintain a low profile. Once they selected the answer, the setting will be applied to all their activities.

Section IV below presents the implemented features of the platform. These features include the overall platform and its architecture, the motivational factors and the usability aspects. It describes a review system that helps users rate a service which will contribute to the Weighted Rating, different usability aspects that give users a sense of control and support, the implemented features to help recognise the contribution of users, and a real-time messaging system to serve the communication need.

\section{RESULT AND DISCUSSION}

\section{A. Result}

The platform has been constructed with the necessary features to facilitate the operation of the platform. Some of the examples are shown below

\section{1) Architecture}

Fig. 1 below shows the overall architecture of the platform. The server side is responsible for serving data on the social network and service network. The client browser handles all the API call with the third-party server and retrieves data such as location and message content. These methods are implemented in JavaScript using jQuery. This approach helps minimize load at the server side.

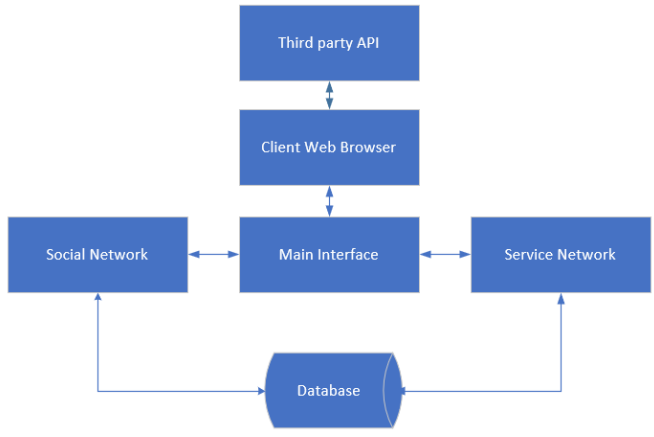

Fig. 1. Overall architecture of the platform

The activities of the users on service network will be shared on their social network. Friends and family in their social connection can help share the service they created and make the service become more popular. As a result, users who would like to maximize the benefit of the service network has a motivation to make more friends. Moreover, when users purchased a service, it creates a notification on 
the social network and allow the service to gain popularity. As the activities of the service network is reflected on social network, the review system plays an important role in determining the success of the service.

Fig. 2 below shows the chat window between users. This feature is implemented on the client side using Google Firebase cloud messaging to provide real-time chat environment. Moreover, Bootstrap is used to ensure the content is displayed correctly on a mobile device (Fig. 6)

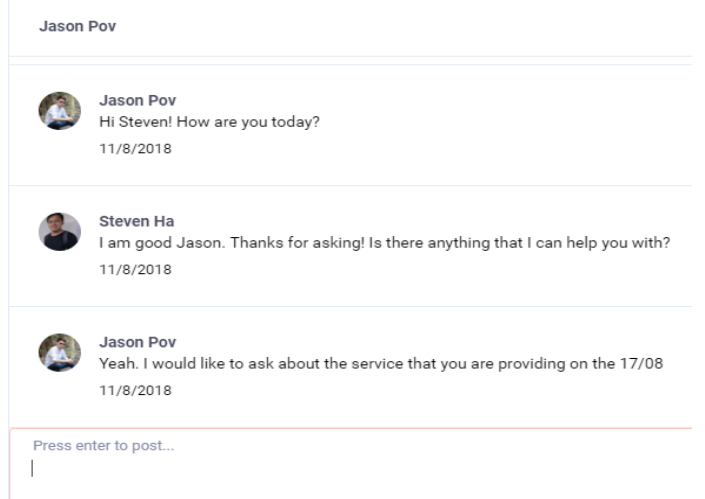

Fig. 2. Chat window using Google Firebase cloud messaging

\section{2) Usability}

Fig. 3 below shows the location of the controls that help users adjust the appearance of the platform. These adjustments include font size and color. Font size is categorized into 3 selections which equivalent to small, medium and large and can be adjusted to suit their needs.

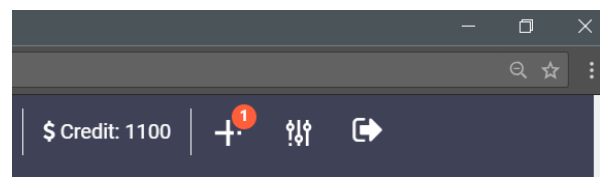

Fig. 3. Location of the controls that help user adjust color and font size

Fig. 4 below shows the different level of color adjustment that is available to users. The choice is ranging from default with a color scheme for those who like bright and high contrast color. Midtone and Soft pastel color for those who may prefer something light and soft. Apart from that, all menu in the platform is designed in such a way that the path of the menu item is wide enough while moving to the submenu. This approach will ensure they can move the pointer down and select the item easily.

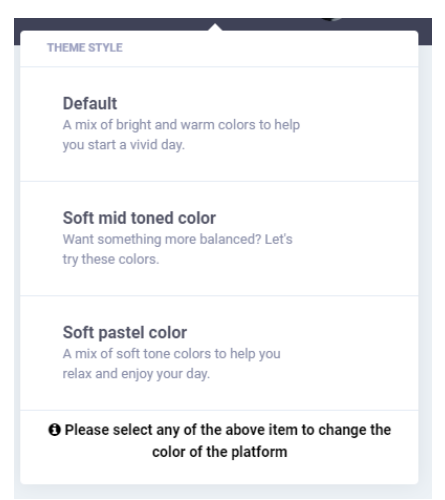

Fig. 4. Different theme selection of the platform
Fig. 5 below shows an example of using tooltip to provide explanation at the field level. Whenever the user clicks on a field, the tooltip will appear indicating the recommended information.

Give your service a meaningful title so people can reate
easily find it
Title

Fig. 5. Example of a tooltip

\section{3) Service network}

Fig. 6 below shows an example of the booking screen of a service on a mobile browser, the upper section shows the details of the service including the provider name, date, time and location of the activity. The lower section allows users to enter their billing details and contact information.

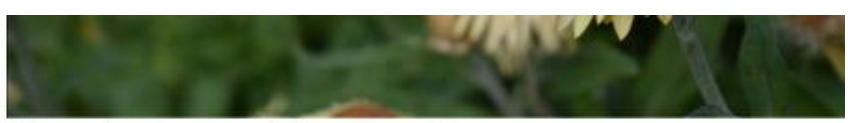

\section{How to grow Chrysanthemums}

\begin{tabular}{|c|c|}
\hline \multicolumn{2}{|c|}{ How to take care of your valuable Chrysanthemums } \\
\hline Location: & New South Wales 2166 \\
\hline Provided by: & Duncan Nguyen \\
\hline Date of activity: & $12 / 08 / 2018$ \\
\hline From: & 9:00 AM \\
\hline To: & 4:00 PM \\
\hline
\end{tabular}

Billing Details

Fig. 6. Example of a booking for a service

Fig. 7 below shows the review of a service on a mobile browser. After the service is delivered, users who booked for the service can review it. They can express their opinion whether the service is good or bad and this factor will be considered by the above algorithm to decide whether this service will be promoted to other users.

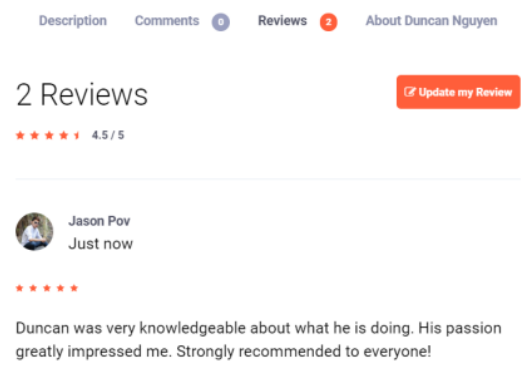

Fig. 7. Example of a review for a service

Fig. 8 below shows the level and achievements of a user based on these achievements, other users can have an overview of the activities that this user participate and determine whether this user is trustworthy before purchasing service from them. A high-level user with many achievements can increase the trust level between users and can result in their service being purchased often. 


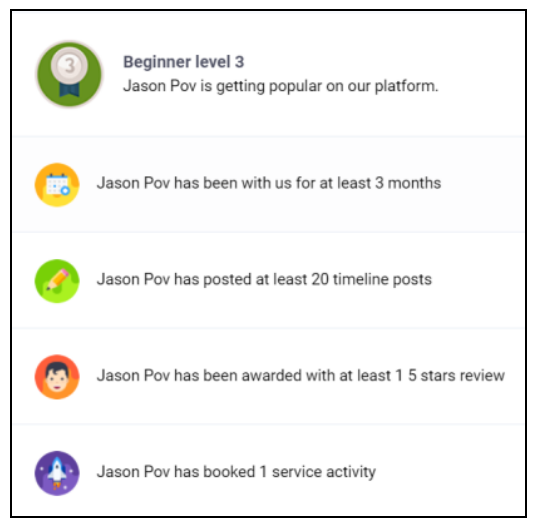

Fig. 8. Example of a user level

The formula is tested with 15 services in different categories with different reviews assigned to them. Based on the above formula, the platform loop through each category and collect the necessary data for calculation to calculate the weighted rating. The top 3 results for each category are selected. If the user does not make specific any interest, the platform promote the top services to them randomly. After they updated their interest, the algorithm selected the services that match their interest and suggest to them.

\section{4) Social network}

The main features to facilitate the social network include

- Adding friends: this feature allows users to expand their social connection by adding other users as friends. Once they are friends, they can see the activities of each other on the platform.

- Messaging: an important feature for both social network and service network, it allows users to exchange information with each other privately.

- Timeline activity: when the service provider hosts a service, it will be updated on their social timeline and their friends can see this activity. As a result, users who would like to make their service popular will need to expand their social connection.

- Profile: This section allows users to update their basic information such as their gender, name, etc. It also contains the level and achievements of that users (Fig. 8 above) which indicates the trust level.

\section{DISCUSSION AND CONCLUSION}

This proposed model outlines a foundation for creating the new type of collaborative platform that allows elderly people to remain active while aging by engaging them to the community. It proposes a solution that serves the needs of seniors by engaging them actively and collaboratively as contributors of services and/or receivers of social benefits. By using the existing technology, the architecture aims to include elderly people in the digital world especially those who are not familiar with smart phone. Moreover, it incorporates many proven principles that are used to engage people together with an interface that not only maximize the accessibility but also give elderly users a sense of control and support. These types of platforms have a vital role in promoting active aging by encouraging elderly people to interact, collaborate and improve mental well-being. For future work, we plan to invite elderly people from the age group of 60 and above to participate in the platform and measure their level of engagement by giving each user a certain point once they registered. The point is calculated as they provide or purchase service. After the study period, users who has more point than they first started indicates that they enjoy providing service to other users. In contrast, users who has less point than they first started indicating that they are using the platform as a place to connect to other people as a service consumer.

\section{ACKNOWLEDGMENT}

This research is supported by an Australian Government Research Training Program Scholarship.

\section{REFERENCES}

[1] Australian Treasury. (2010). Australia to 2050: Future challenges [Online]. Available http://archive.treasury.gov.au/igr/igr2010/Overview/pdf/IGR_2010_O verview.pdf

[2] Australian Bureau of Statistics (2008). Australian Historical Population Statistics [Online]. Available at: http://www.abs.gov.au/AUSSTATS/abs@.nsf/DetailsPage/3105.0.65. 0012008?OpenDocument

[3] Productivity Commission. (2013). An Ageing Australia: Preparing for the Future [Online]. Available at: https://www.pc.gov.au/research/completed/ageing-australia/ageingaustralia-overview.pdf

[4] ABC (2015), Why are elderly Australians taking their own lives? [Online]. Available at: <http://www.abc.net.au/news/2015-1008/mental-health-depression-elderly-suicide/6808998>.

[5] J. Coelho, C. Duarte, "A literature survey on older adults' use of social network services and social applications," Computers in Human Behavior, vol.58, pp. 187-205, 2016.

[6] S.S. Sundar, A. Oeldorf-Hirsch, J. Nussbaum, R. Behr, "Retirees on Facebook: can online social networking enhance their health and wellness?," in CHI '11 Extended Abstracts on Human Factors in Computing Systems, Vancouver, BC, 2011, pp. 2287-2292.

[7] R. Cornejo, N. Weibel, M. Tentori and J. Favela, "Promoting active aging with a paper-based SNS application," in CHI '11 Extended Abstracts on Human Factors in Computing Systems, Vancouver, BC, 2011, pp. 2287 - 2292.

[8] W.C. Fang, M.C. Hsieh, P.C. Yang, W.G. Li, C.J. Chiu, J.H. Chiang, "iDianNao: an orange technology that recommends volunteer opportunities to older adults," in 2015 International Conference on Orange Technologies (ICOT), Hong Kong, 2015, pp. 38-41.

[9] N. Morrow-Howell, J. Hinterlong, P. A. Rozario and F. Tang, "Effects of volunteering on the well-being of older adults," The Journals of Gerontology Series B, vol. 58, no. 3, pp. S137-S145, 2003.

[10] H. Pensas, T. Kivimäki, A. Vainio , S. Konakas, S. Costicoglou, P. Kölndorfer, K. Summanen, H. Moisio and J. Vanhala, "Building a Client-Server Social Network Application for Elders and Safety Net," in AcademicMindTrek '13 Proceedings of International Conference on Making Sense of Converging Media, Tampere, Finland, 2013, pp. 310-312.

[11] I. Marcelino, R. Laza and A. Pereira, "SSN: Senior Social Network for Improving Quality of Life," International Journal of Distributed Sensor Networks, vol. 12, 2016.

[12] T. V. Ha, D. B. Hoang, "An assistive healthcare platform for both social and service networking for engaging elderly people," in 2017 23rd Asia-Pacific Conference on Communications (APCC), Perth, WA, Australia, 2018, pp. 1-6

[13] M. Callow, "Identifying promotional appeals for targeting potential volunteers: an exploratory study on volunteering motives among retirees," International Journal of Nonprofit and Voluntary Sector Marketing, vol. 9, pp. 261-274, 2004.

[14] A. M. Sellon, "Recruiting and Retaining Older Adults in Volunteer Programs: Best Practices and Next Steps," Ageing International, vol. 39, pp. 421-437, 2014. 
